\title{
Partner Notification Services (PNS) Among HIV Positive Key Populations: An Important Strategy Towards Achieving UNAIDS 95.95.95 Goals by the Year 2030
}

\author{
Ugbena, Eneojo Richard $^{1 *}$, Egbe, Aneaton ${ }^{1}$, Ikani, Samuel ${ }^{1}$, Okekearu, Ifeanyi ${ }^{1}$ \\ ${ }^{1}$ Society for Family Health, Nigeria
}

\begin{abstract}
Key Populations are the major drivers of the HIV epidemic in most settings; thus, identifying positive KPs and their sexual partners becomes a strong strategy in the effort to achieve the first UNAID 95 goal. This study describes the level of acceptability and outcome of PNS among HIV-positive KPs using the various approaches for PNS. A descriptive study of all HIV positives KPs initiating ART in 7 OneStop-Shop facilities that accepted PNS was conducted over 3 months. With the consent of patients who accepted PNS, their partners were reached using any of the PNS approaches. Partners who consented for HTS were tested, and positives partners were linked to ART services. Data were analyzed to describe the acceptability and outcome of PNS among KPs. Only 846/2,486 (34\%) positive KPs accepted PNS. The 846 KPs elicited 941 partners, out of which 938 (99.6\%) accepted HTS. 421/938 (45\%) of the partners tested positive compared to PWIDF (15\%), MSM (7\%), FSW (4\%) and PWIDM (4\%). 129/421 (31\%) of the positive partners were already known positives, with $61 \%$ already on ART. The commonest method of PNS accepted among KPs was the passive method (54\%), followed by the contract method (22\%), provider-initiated (19\%), and dual method (5\%). There was poor acceptance of PNS among KPs and, the HIV positivity rate was much higher among their partners than any of the KPs sub-groups. The passive approach was the most preferred method of PNS among KPs. This should be considered strongly as we scale up PNS in Nigeria.
\end{abstract}

Keywords: HIV/AIDS, Key Populations in Nigeria, Partner notification services, UNAIDS 95-95-95 targets.

\section{Introduction}

It was estimated that, as at the end of 2015, over 36 million people worldwide had HIV, and of these, $40 \%$ remained undiagnosed [1]. This figure increased to approximately 38 million people in 2019 across the globe, of which only $81 \%$ knew their HIV status [2]. In Nigeria, only $34 \%$ of the adult population knew their HIV status as of 2016 [3]. The majority of the world's new HIV infections occur in resource-poor countries, with two-thirds of the world's HIVinfected population living in Africa [4]. Nigeria has the second-largest HIV epidemic burden in the world, second to South Africa [5]. Although Nigeria HIV prevalence among adults is $1.5 \%$ compared to other sub-Saharan African countries such as South Africa (19\%) and Zambia (11.5\%), the size of Nigeria's population means that about 1.9 million people were living with HIV in 2019 [5]. The country has a mixed epidemic, meaning that while HIV prevalence among the general population is high, certain groups still carry a far greater HIV burden compared to the rest of the population [5]. Also, from previous sentinel surveys, the distribution of HIV across all states is not uniform. While some states have a very high prevalence above the national average, others have very low prevalence compared to the national average [6]. In most countries and settings, Key Populations (KPs) are disproportionately affected by HIV 
infection. KPs are groups that have a high risk and disproportionate burden of HIV in all epidemic settings due to their behavioral patterns and specific legal and social challenges that increase their vulnerability to HIV, including barriers to accessing HIV prevention, treatment, and other health and social services [7]. Key populations include Men who have Sex with Men (MSM), People Who Inject Drugs (PWID), and Brothel Based Female Sex Workers (BBFSW), None Brothel Based Female Sex Workers (NBBFSW), people in prisons and closed settings, and transgender people. In Nigeria, the prevalence of HIV among MSM, BBFSW, NBBFSW and PWID were 23\%, $19.4 \%, 8.6 \%$ and $3.4 \%$ respectively. [8] compared to the national prevalence of 3.0 as at 2014 [6]. These sub-populations; Sex workers, men who have sex with men, and people who inject drugs, make up only $3.4 \%$ of the population, yet account for around 32\% of new HIV infections [5]. The implication is that these sub-populations will continue to drive the epidemic within the country even though there has been a general decline in prevalence among KPs from 2010 to 2014 except for MSM who have shown a consistent increase from $14 \%$ in 2007, $17.2 \%$ in 2010 and $22.9 \%$ in 2014 [9]. Because of stigma and discrimination and the law criminalizing high-risk behaviors among KPs in Nigeria, most KPs do not identify as KPs thus, endangering the general public. KPs continue to drive the HIV epidemic because sexual transmission by KPs is not limited within the groups. Program experience has shown that an MSM may be married to a female partner without the wife knowing that her husband is an MSM. The clients of FSW are from the general population some who are regarded as special boyfriends and usually does not use a condom to prevent HIV transmission.

Similarly, some of the PWID usually get married or are engaged in sex works thus, and they have the dual risk of the method of infection. Besides, because of their sexual orientation, KPs generally have poor health- seeking behavior due to stigma and discrimination at health facilities and in the general public. These practices obviously, fuels the continuous spread of HIV infection among KPs and the general population.

The World has continued to make an effort on the control and elimination of HIV/AIDS. The Joint United Nations Program on HIV and AIDS (UNAIDS) has been the main advocate for accelerated, comprehensive, and coordinated global action on the HIV/AIDS pandemic since 2001 [10]. In 2011, based on achievements made at the time, UNAIDS set a global target of putting 15 million HIV/AIDS patients on treatment by 2015 . The " 15 by 15 " target was adopted at the United Nations High-Level Meeting in 2011, as part of the political declaration on HIV/AIDS: "Intensifying Our Efforts to Eliminate HIV and AIDS" [11]. The target of 15 million people receiving HIV treatment by 2015 was reached nine months early-proof of the global desire and commitment to eliminating the HIV/AIDS epidemic.

The lessons learned in the successful global push to provide antiretroviral therapy to 15 million people by 2015 provided a roadmap for ending the AIDS epidemic as a public health threat. As a central component of the effort to end the epidemic, UNAIDS came up with a new target called UNAIDS 90-90-90 for antiretroviral therapy to be achieved by 2020 [11]. This target is an intermediate goal towards achieving an ambitious goal of reaching 95-9595 by the year 2030 to end the HIV/AIDS epidemic. The 90-90-90 targets imply that by $2020,90 \%$ of all people living with HIV will know their HIV status, $90 \%$ of all people with an HIV diagnosis will receive sustained antiretroviral therapy, and $90 \%$ of all people receiving antiretroviral therapy will achieve viral suppression [11]. These new developments were described as "game changers," adding new tools to a long-established mix of behavioral, biomedical, and structural HIV prevention interventions [12]. According to UNAIDS, if the 
new target could be achieved, the number of new HIV infections will be reduced to under 500000 in 2020 and under 200000 in 2030, thus effectively ending the AIDS epidemic as a public health threat. Compared with a 2010 baseline, these numbers would constitute a $75 \%$ reduction in new HIV infections by 2020 and a $90 \%$ reduction by 2030 [13]. Since they were launched at the 20th International AIDS Conference in Melbourne, Australia, in 2014, the 90-90-90 targets became a central pillar of the global quest to end the AIDS epidemic. The targets reflect a fundamental shift in the world's approach to HIV treatment, moving it away from a focus on the number of people accessing antiretroviral therapy and towards the importance of maximizing viral suppression among people living with HIV [14].

Four years after the launching of UNAIDS 90-90-90, global leaders from civil society, governments, the private sector, and academia came together for a two-day workshop on 21 and 22 July in Amsterdam, Netherlands, to highlight the successes, identify gaps and share best practices in order to reach 90-90-90 [15]. By the end of 2017, the world had achieved 75-79-81, meaning that globally, $75 \%$ of people living with HIV knew their status, $79 \%$ of people living with HIV who knew their status were accessing antiretroviral therapy, and $81 \%$ of people accessing treatment had suppressed viral loads [15].

According to UNAIDS estimates, there were around 36.9 million people living with HIV worldwide in 2017, with around 21.7 million people accessing life-saving medicines that keep people alive and well and stop the transmission of the virus, about 1.8 million new HIV infections occurred while 940000 people died from AIDS-related illness worldwide, compared to 1.9 million in 2004 [16]. However, UNAIDS also estimates that AIDS-related deaths have been reduced by more than $51 \%$ since the peak in 2004, but around one in four people worldwide continue to be unaware that they are living with HIV [16].
Despite the global successes, the evidence presented at a workshop in Amsterdam showed that entire regions and populations are still being left behind. Progress in eastern Europe and central Asia, western and central Africa, and the Middle East and North Africa were falling behind [15]. Data from a variety of sources showed that gaps in the 90-90-90 continuum are greater for key populations, men and young people, [14] because they were not being reached by traditional health facility-based HIV testing services. Lack of political commitment, user fees, and stigma and discrimination were some of the identified barriers to progress [15]. Other barriers are [14]: harmful masculine gender norms which contributed to greater risktaking and poorer uptake of health services among men; consent laws and insufficient access to comprehensive sexuality education which denied young people the services and knowledge they need; Key populations often face criminalization and high levels of stigma and discrimination. Thus, closing gaps in service coverage requires intensified efforts to convince men to reject harmful versions of masculinity and to reach and empower women and girls, young people, and key populations, to enhance their agency and to ensure their human rights are respected and protected. Also, addressing stigma, discrimination, and human rights violations at all levels through the creation of a protective and empowering legal environment and the strong rule of law is imperative for both the AIDS response and the wider 2030 Agenda for Sustainable Development [14].

As of 2019, a review of the progress made on achieving the UNAIDS 90-90-90 goal showed that 14 countries had met the targets [17]. However, in June 2020, the UNAIDS report on the global AIDS epidemic showed that 2020 targets could not be met because of deeply unequal success within and between countries with COVID-19 risks blowing HIV progress way off course [17]. With the 90-90-90 targets unachievable in 2020, the new effort is geared towards doubling our effort towards the 
attainment of the UNAIDS 95-95-95 targets in 2030 using the fast-track approach [18]. The Fast-Track approach is an agenda for quickening the pace of implementation, focus, and change at the global, regional, country, province, district, and city levels. It involves setting ambitious targets and accelerating the delivery of highimpact HIV prevention and treatment services. It means using innovation to expand services, to better address people's needs and perspectives, and focus on the locations and populations with the highest HIV burden. It addresses social and legal barriers and advances human rights and gender equality.

To be able to address the gap in knowledge of HIV status, which is the first target in UNAIDS 95-95-95 goals, will require a new approach that will enhance the efficiency and coverage of HIV testing both in the general population and among KPs. HIV partner notification is an approach that has the potential to improve coverage while also identifying people with undiagnosed HIV infection [1] and one of the most important ways to prevent the spread of HIV [19]. HIV partner notification is a voluntary process where trained health workers, including lay providers, ask people diagnosed with HIV about their sexual partners or drug-injecting partners, and with the consent of the HIV-positive client, offer these partners voluntary HIV testing [1][20]. Usually, the person diagnosed with HIV is encouraged to voluntarily disclose their status and notify their partner(s). Their partner(s) can then be confidentially contacted and offered voluntary HIV testing services, prevention, treatment and care if they test positive [21]. These services will ensure that persons in a sexual network are identified to access HIV services as early as possible, even before the manifestation of the disease. PNS can be offered either actively or passively. While passive notification means that the person diagnosed with the infection voluntarily notify their partner, but in an active notification, the positive patient gives consent to the health care worker to assist in whichever way possible to notify their partner. Once an index case has identified a partner, they should be notified as soon as possible. This will give the partner an opportunity to protect themselves from infection if they don't have HIV or, if they are having sex or sharing needles with others, to take steps to protect or notify those partners [19].

Active or assisted partner notification has been an important public health approach in infectious disease management for decades, including programs targeting sexually transmitted infections (STIs) and tuberculosis (TB) [1]. STI partner notification approaches have been shown to be effective in diagnosing and treating STIs and preventing recurrent infection.

Likewise, active tracing of contacts and the voluntary screening of household members of people with active TB is an effective and standard approach that has been used successfully in communities with high HIV and TB prevalence. In 2012 WHO developed guidance recommending couples and partner HTS, including support for a mutual disclosure, with a special focus on testing the partners of people diagnosed with HIV infection in all epidemiological settings [1]. The benefits of partner and couples HTS have been well documented, including mutual support to access prevention, treatment, and care services, as well as improved adherence and retention in treatment and prevention of mother-to-child transmission programs. Partner testing also allows those in serodiscordant partnerships to prioritize effective HIV prevention, such as the use of condoms, immediate antiretroviral therapy (ART), medication adherence by HIVpositive partners, and pre-exposure prophylaxis (PrEP) for HIV-negative partners.

Because the sexual partners of HIV positive individuals have an increased probability of also being HIV positive if they continue to have sex without taking any of the prevention measures, it, therefore, become a very important for partners of positive KPs to know their HIV status and take appropriate measures depending on whether they turn out positive or negative. This 
implies that at the point of diagnoses or when the KP knows their HIV-positive status, they must have the opportunity to identify their partners who are likely to be at risk of contracting the infection from them. However, partner testing services, including partner notification, for people diagnosed with HIV have not been routinely offered or implemented; therefore, uptake and coverage remain low [1]. For KPs who are drivers of the epidemics in all settings, it is important to offer PNS to all positives KPs in order to elicit all their sexual partners who are at risk of contracting HIV infection.

This process will help to identify HIV positives cases among persons in the sexual network early enough and put them on treatment. Previous studies [22, 23, 24] have shown that the early HIV-positive persons are identified, linked to treatment, and retained on treatment, the earlier they will achieve viral load suppression and, thus, reduced rate of transmission to uninfected persons. This is the idea behind the UNAIDS 95-95-95 goals that if $95 \%$ of persons who are infected know their HIV status, 95\% of those who know their HIV status are linked and retained on treatment and, if $95 \%$ of those retained on treatment achieve viral load suppression by 2030 then, HIV/AIDs epidemic would have been controlled. The PNS among KPs remains very relevant as a recent report by UNAIDS in 2020 showed that there is unequal progress in the fight against HIV/AIDS, with too many vulnerable people and sub-populations as KPs are left behind.

A study has described that about $62 \%$ of new HIV infections occurred among key populations and their sexual partners, including gay men and other men who have sex with men, sex workers, people who inject drugs, and people in prison, despite them constituting a very small proportion of the general population [17]. Therefore, it is very important to implore any strategy like PNS among HIV positives KPs to be able to identify their partners who are positive and link them to treat to be able to achieve viral suppression and limit further transmission.
The object of this study was to describe the level of acceptability and outcome of PNS among HIV positive KPs using the various approaches for PNS services and to describe the proportion of HIV positive KPs who accepted partner notification services after diagnosis. This work also defines the various methods of partner notification services used to reach the partners of Key Population and describe the proportion of KPs partners who accepted HIV testing after notification. This study is to determine the HIV prevalence among partners of KPs who consented and tested for HIV.

\section{Methodology}

\section{Study Area}

This study was carried out across 7 states supported by SHiPS for the MARPS project in Nigeria.

In each of the states, the project established a One-Stop-Shop facility to provide HIV prevention, treatment, and care services for Key Populations and their partners. Other services provided at the sites include partners notification services, syndromic management of STIs, and cervical cancer screening.

\section{Study Design}

This was a descriptive study using routine program data. All HIV-positive KPs accessing services in the 7 One-Stop-Shop facilities were offered PNS during enrollment into a treatment program.

Those who consented chose one of the approaches of partner notification services, and effort was made by the project to reach their partners. Consent was obtained from the partners who were not previously known positives to take HIV screening, and all partners who tested positives were linked to treatment services.

\section{Sampling Technique}

There was no sampling of participants, but all HIV positives KPs identified at the 7 OSS across the 7 states during the period of study were offered PNS. KPs who consented for PNS were 
enrolled and were asked to provide the list of their partners to be notified. The participants also agreed with the health care providers on the best approach to reach their partners. Voluntary counselling and testing for HIV was provided to partners who were notified and accepted HIV testing.

\section{Data Collection Methods}

A partner notification services register (a PEPPFAR tool) was used to capture data on participants, their partners, and the outcome of HIV testing among partners as part of routine program services. Data was abstracted from the routine service register by the facility record clerk who was trained on data abstraction for the study.

\section{Ethical Consideration}

Only participants who accepted PNS were enrolled for the study, and participant's records were identified using PNS unique identification number. The researcher did not meet the participants in person neither was their names or location included in the data abstracted. Only partners who consented for HIV testing after notification were provided voluntary counselling and testing and provided results. There was no disclosure of the participant who enlisted a partner and verse versa, and neither was there a disclosure of the outcome of the testing result of the partner to the participants who enlisted them except if there was consent to do so.

\section{Study Limitation}

The study did not elicit reasons why clients declined PNS. With the low acceptance rate of PNS, it is important to know various reasons why clients did not accept PNS. This is important for the program to be able to strategize on improving the acceptance rate. Also, there was no data to show which of the KP sub-group has the highest PNS acceptance rate, what method of PNS was mostly preferred by subpopulations of KP, and the group that has the highest positivity rate among their partners. Further study should include these finer analyses to provide more information to guide programming.

\section{Results}

Table 1. PNS Cascade Among Participants

\begin{tabular}{|l|l|l|}
\hline PNS cascade & Number & Percent \\
\hline Number of KPs enrolled into the program during the period & 47668 & \\
\hline Number of KPs who received HIV counselling and testing & 47668 & $100 \%$ \\
\hline Number of KP who tested positive & 2486 & $5 \%$ \\
\hline Number of HIV positive KPs who accepted PNS & 846 & $34 \%$ \\
\hline Number of Partners elicited by HIV positive KPs & 941 & \\
\hline Number of partners notified and tested for HIV & 938 & $99.60 \%$ \\
\hline Number of KPs partners who tested HIV positive & 421 & $45 \%$ \\
\hline
\end{tabular}

Out of 47,668 KPs tested during the period, 2,486 (5\%) tested HIV positive, with only 846 (34\%) accepting PNS. The 846 positive KPs elicited 941 partners, out of which 938 (99.6\%) were notified and accepted HIV counselling and testing. Four hundred and twenty-one (45\%) of the partners who tested for HIV were positive for the virus. 
Table 2. Sex and HIV Prevalence among Participants

\begin{tabular}{|l|l|l|l|l|}
\hline Sex & Number tested & $\boldsymbol{\%}$ & Number pos & Prevalence \\
\hline Male & 9534 & 20 & 895 & $9.3 \%$ \\
\hline Female & 38134 & 80 & 1591 & $4.1 \%$ \\
\hline Total & 47668 & 100 & 2486 & \\
\hline
\end{tabular}

Twenty percent of the participants were male, which included MSM, PWIDM, and male partners, while the remaining $80 \%$ were female, made up of FSW, PWIDF, and female partners. HIV positivity rate was higher among males 9.3\% than among females $4.1 \%$.

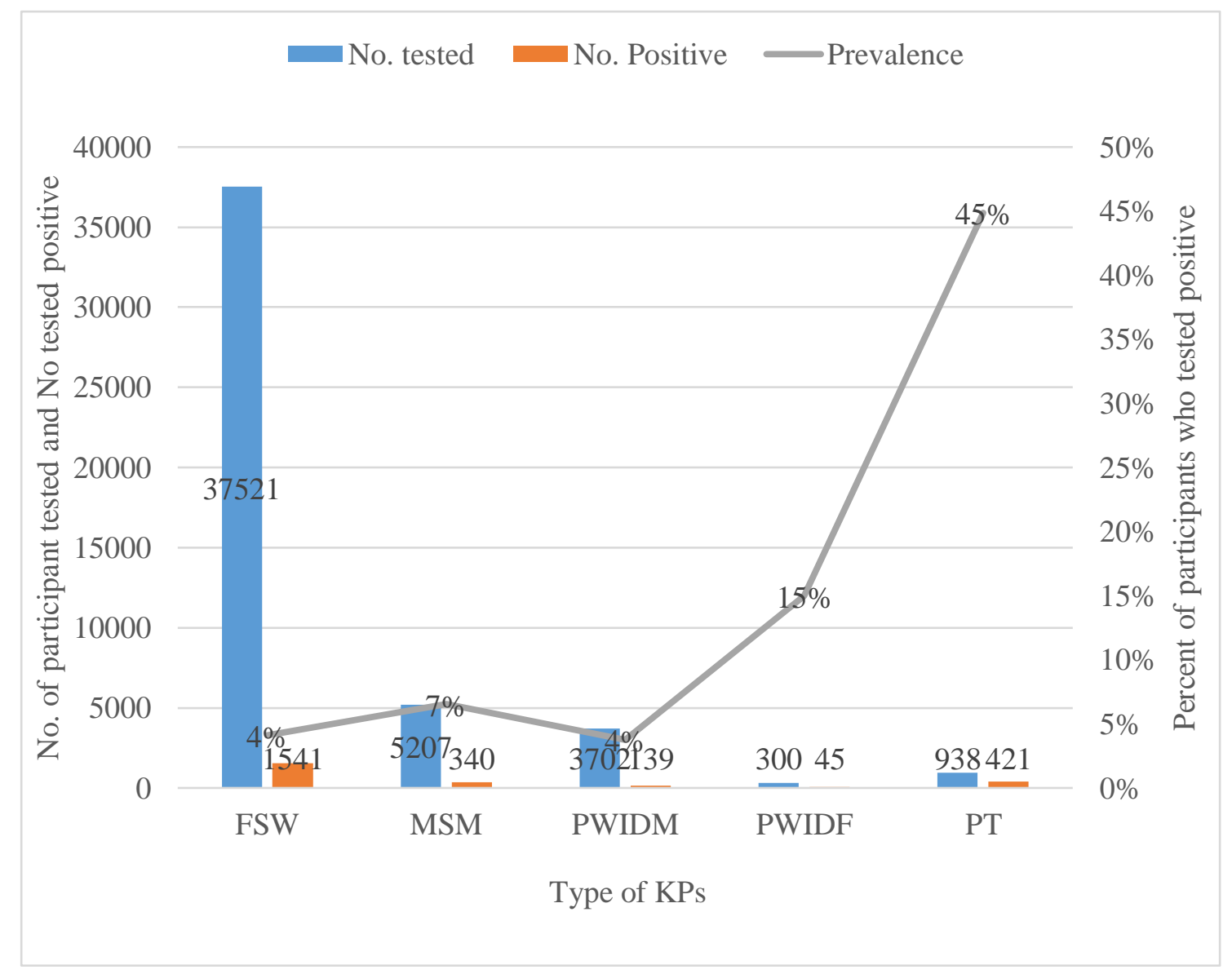

Figure 1. HIV Prevalence among KPs and their Partners 


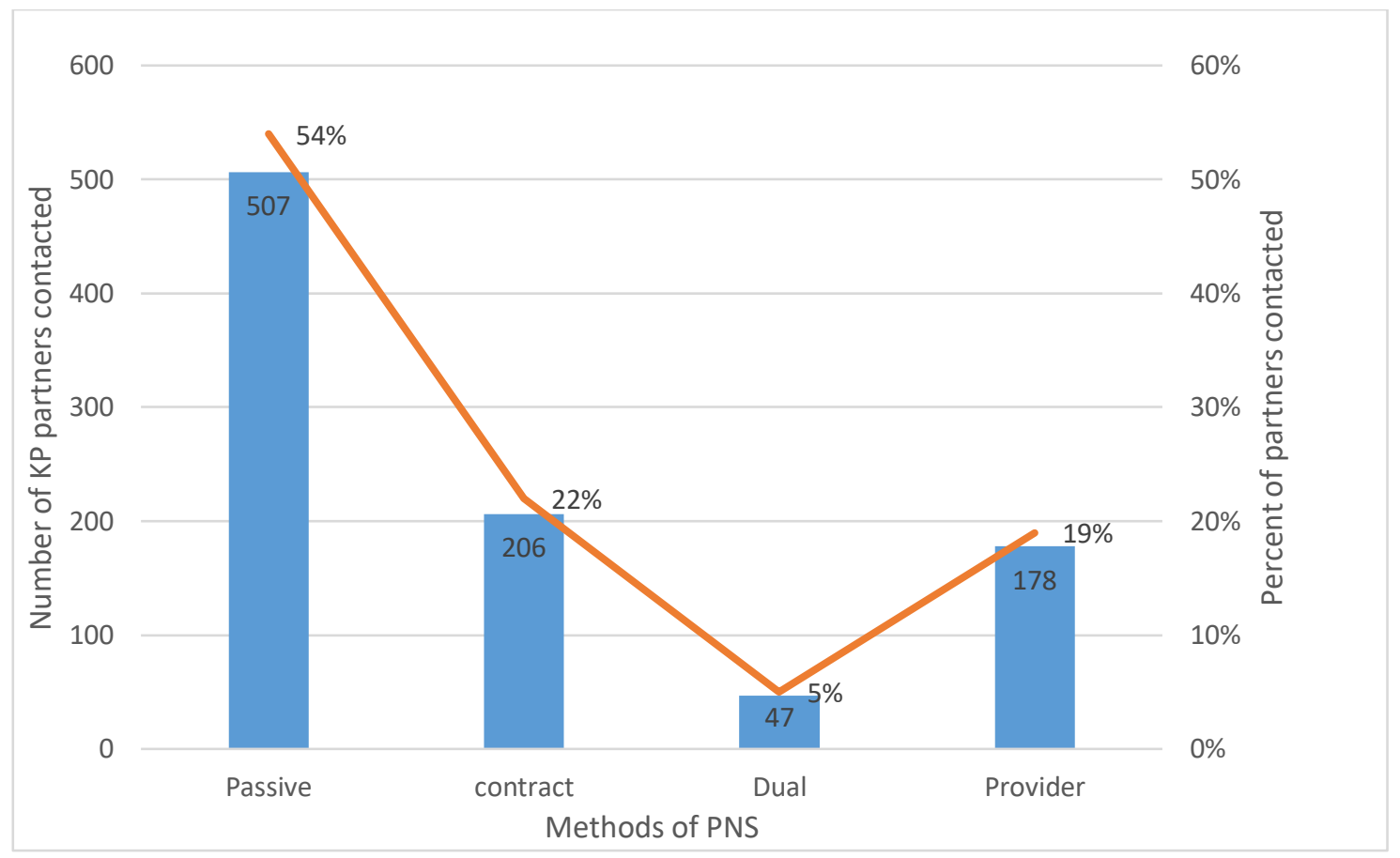

Figure 2. Methods of Partner Notification Services

The commonest method of partner followed by contract method (22\%), providernotification accepted by KPs was a passive initiated (19\%), and dual method was the least method which accounted for $54 \%$ of cases, method (5\%).

Table 3. HIV Positive Partners of KPs and Status of ART Services at the Time of Partner Notification and Testing

\begin{tabular}{|l|l|l|l|}
\hline \multicolumn{4}{|l|}{ ART status } \\
\hline HIV status & On ART (\%) & Not on ART (\%) & Total (\%) \\
\hline Known Positive & $77(61 \%)$ & $52(39 \%)$ & $129(31 \%)$ \\
\hline New positive & $0(0 \%)$ & $292(100 \%)$ & $292(69 \%)$ \\
\hline Total & $77(18 \%)$ & $344(82 \%)$ & $421(100 \%)$ \\
\hline
\end{tabular}

Out of the 421 partners who tested positive, $129(31 \%)$ were previously known positive, while 292 (69\%) were new positive cases identified. Of the 129 who were already known positives, $61 \%$ were already on ART, while the remaining $39 \%$ are ART naïve and were linked to ART services. Overall, $82 \%$ of HIV positive clients identified needed to be linked to HIV care and treatment services.

\section{Discussion}

The acceptance of HIV testing among the general population in Nigeria was $76 \%$ [25], while it varies among KPs: PWID 54\%, MSM
65\%, and FSW 88\% [8]. However, in an organized program such as provider-initiated counselling and testing, previous studies have demonstrated a higher acceptance rate of $94 \%$ [26] and $98.7 \%$ in Nigeria [27]. Similarly, on this project, "strengthening HIV prevention services for Most at Risk Populations where HIV counselling and testing is part of the intervention, $100 \%$ of the participants accepted testing, and those who tested positive were linked to treatment services.

Although Nigeria has a generalized HIV epidemic, the spread is not the same across all 
the regions, states, and even within the same state. The NAIIS survey reported a national prevalence of $1.4 \%$, with the prevalence generally higher among females than males and more among the youth than older adults [28]. This new report shows a lower burden of HIV infection in the country compared to previous surveys that reported prevalence of 3\% [6]. Also, previous surveys among Key Populations have shown varied burdens with MSM having the highest prevalence of $23 \%$, BBFSW $19.4 \%$, NBBFSW 8.6\%, and PWID 3.4\% [8] compared to the finding in this study which shows a prevalence of $7 \%$ for MSM, $4 \%$ for FSW, PWID male $4 \%$, and PIWD female $15 \%$. The higher prevalence reported by the survey may be related to the methodology for recruiting participants, which used snow-ball sampling and financial inducement for the index case based on the number of participants recruited by an index case. The snow-ball method and financial benefit to index case have the tendency for a positive KP to recruit another positive $\mathrm{KP}$ compared to the project intervention, which enrolled participants by visiting the location of KPs and testing all who consented. Besides, the project also delivered HIV prevention services for cohorts of KPs within the communities as part of the intervention strategy, which raised awareness on HIV prevention among the KPs, resulting in lower prevalence among the intervention group. The higher prevalence among PWID females than PWID males may be due to the dual risk of infection among PWID females who are mainly FSW with multiple sexual partners and sharing unsterilized needles with fellow drug users. Also, since most of the PWID females are FSW, the effect of drug use increased their vulnerability to high sexual risk behavior. The overall prevalence of 5\% among KPs in this study compared to $1.4 \%$ [28] among the general population implies that Key Populations will continue to drive the epidemic since their sexual network cut across both Key Populations and the general populations. For the world to meet up with the UNAIDS 95-95-95 goals requires that most infected persons must be identified, linked, and retained on treatment to be able to achieve viral suppression and the ultimate benefit of a reduced new infection. A very significant finding in this study is the 421/938 (45\%) HIV prevalence among partners of KPs who consented, tested for HIV, and received their test results. Similar intervention among the general population in Nigeria. [29] reported a prevalence of $51 \%$. Various rates have also been reported for other countries, demonstrating a very high positive yield from PNS. Out of the 421 positives identified, $77(18 \%)$ were known positives already on ART, while the remaining $82 \%$ were either newly identified cases or old cases that were not linked to ART services. The finding from this study has demonstrated that HIV-positive case finding using PNS services among KPs is very effective in reaching sexual networks of the drivers of the epidemic in a country where sexual transmission is the major mode of spreading the disease. Without PNS services, the 344/421 (82\%) new positives cases will be left in the community and may continue to spread the infection. Overall, PNS is a necessary strategy that we must use for both the general population and Key Populations if we must achieve UNAIDS first "95" of the 9595-95 goals.

An important concern found in the study is the PNS acceptance rate by HIV-positive KPs. Only $34 \%$ of the positive cases accepted that their partners should be notified of their risk for acquiring HIV through them. This is one of the challenges earlier described by a study [30] amongst KPs who were less able or willing to identify partners. Although this also occurred among the general population, particularly among people with casual partners, recall of and contact information for partners was better among heterosexual women than among men who have sex with men or people who inject drugs [30]. Such a low acceptance rate will translate to a missed opportunity for a high 
positive yield that has been described for PNS. It is imperative that programs will need to do more to be able to overcome such challenges in order to reap the benefit of PNS. However, it was quite impressive to see that almost all partners (99.6\%) who were notified consented for HIV testing and were tested and received their test result with a $45 \%$ positivity rate. A much higher $72 \%$ positivity rate has been reported among partners of HIV-positive pregnant women, while among PWID and MSM, the rates vary from 5$80 \%$ [30]. This high positivity rate among sexual partners implies that transmission is largely through sexual networking.

In this study, the HIV prevalence among male participants is $9.3 \%$ higher than $4.1 \%$ among females, even though in the general population, the prevalence is higher among females than males. This may be due to the high prevalence among MSM partners, and besides, most partners of FSW are male, resulting to a larger pool of males tested among the partners of KPs. Particularly at a point when an effort is being made to get more men for testing, PNS has shown how testing and positivity yield can be improved among men.

The most acceptable method of partner notification services in this study is the passive method, where the positive index cases are expected to notify their partner themselves of their HIV positive status and to encourage their partners to take HIV tests to determine their own status. This is contrary to most studies in [30], where assisted/provider-initiated method is preferred. However, a study in Tanzania [30] has reported a high rate $(93 \%)$ of acceptance of passive notification among participants overassisted methods. Similarly, in a study among FSW in Guatemala, $85 \%$ of the women preferred passive notification over letter or phone call by a health provider [30]. In a study in the US among MSM, assisted PNS was preferred because they believe that it protected against blame, violence, and stigma. In Singapore, men who have sex with men preferred email for partner notification significantly more than did heterosexual respondents. The choice of passive method over the contract and provider-initiated methods may be related to the fear that their partner may not be comfortable with the presence of the third party during the notification process. Although more success rate has been reported through the provider-initiated method in other settings, the higher acceptance rate of 54\% among partners using the passive method in this study has also demonstrated that the passive method may be preferred by the KP subpopulations perhaps due to high level of stigmatization, discrimination, and criminalization of KPs in the country. Also, the higher acceptance rate of $46 \%$ for assisted PNS implies that no one method is preferred. Thus, various options of PNS should be provided to any client in order to improve the acceptance rate among index cases.

\section{Conclusion}

Acceptance of PNS among KPs is very low, but the prevalence of HIV is much higher among partners who were identified and tested than the KPs sub-groups.

Most KPs preferred a passive approach to notify their partners of their HIV positive status and then encourage them to determine their own status.

For an effective HIV control in 2030 as recommended by UNAIDS, targeted testing through PNS is key to identifying a lot of HIV positives individual who will be missed if HIV interventions target only people who identifies as KPs. The low acceptance of PNS among KPs is a challenge for HIV intervention. More evidence is required to identify an innovative way to reach partners of HIV positives KPs, encourage them to determine their HIV status, and take necessary steps for HIV prevention, treatment, or care services as may be necessary.

\section{Recommendation}

1. As we scale up PNS in Nigeria, this study has shown that passive referral is the most 
acceptable approach among KPs, and this should be considered strongly in programming PNS among KPs.

2. Only $34 \%$ of the KPs accepted PNS. There is the need for strong counselling at the point when PNS is offered to stress the benefit of PNS. This is likely to improve PNS uptake among KPs.

3. There is a need for policy formulation that will create enable environment for PNS activities. Law enforcement agents and legal practitioners should not treat issues of PNS as a crime.

4. Because both passive and assisted methods of PNS are highly rated in this study, any index case should be allowed to choose any of the methods he or she deem appropriate for each partner.

5. One limitation of this study is that there is no data to show which of the KP sub-group has the highest acceptance of PNS and the subgroup that has the highest positivity rate

\section{References}

[1] World Health Organization Geneva (2016). Guidelines on HIV Self-Testing and Partner Notification: Supplement to Consolidated Guidelines on HIV Testing Services: World Health Organization; 2016 Dec. 3, Available from: https://www.ncbi.nlm.nih.gov/books/NBK401676/.

[2] The Global HIV epidemics (2019). Available at https://www.hiv.gov/hiv-basics/overview/data-andtrends/global-statistics.

[3] Averts (2020). HIV and AIDS in Nigeria. Available at https://www.avert.org/professionals/hivaround-world/sub-saharan-africa/nigeria.

[4] Jennifer L. Weinberg and Carrie L. Kovarik MD. (2010). The WHO Clinical Staging System for HIV/AIDS, American Medical Association Journal Ethics, Volume 12, Number 3: 202-206.

[5] Averts (2020). HIV and AIDS in Nigeria. Available at https://www.avert.org/professionals/hivaround-world/sub-saharan-africa/nigeria. among their partners. Further study should include this finer analysis to guide programing.

\section{Acknowledgement}

The authors acknowledge the Key Populations members who participated in the study and the OSS staff for the incredible work and dedication to duty that ensured accurate data was abstracted for the study. None of the authors has any conflict of interest to declare.

\section{Funding}

The SHIPS for MARPS project were funded by USAID through President's Emergency Funds for AIDS Relief (PEPFAR) and implemented by Society for Family Health, Nigeria.

\section{Conflict of interest}

There authors have declared that there is no conflict of interest in carrying out this study.

[6] Federal Ministry of Health Nigeria (2015). HIV Sero-prevalence among pregnant women in sentinel antenatal clinics by states: Available at http://www.sfhnigeria.org/wpcontent/uploads/2018/05/National-Strategic-framework-Final-28th-May-2018.pdf.

[7] World Health Organization (2014); Consolidated guidelines on HIV prevention, diagnosis, treatment, and care for key populations. Available at http://apps.who.int/iris/bitstream/10665/128048/1/97 89241507431_eng.pdf?ua=1\&ua=1.

[8] Federal Ministry of Health Nigeria (2015). Integrated Biological and Behavioural Sentinel Survey (IBBSS 2014). Available at https://naca.gov.ng/wpcontent/uploads/2016/11/Final-Nigeria-IBBSS-

2014-report.pdf.

[9] Eluwa, G.I., Adebajo, S.B., Eluwa, T. et al. (2019). Rising HIV prevalence among men who have sex with men in Nigeria: a trend analysis. $B M C$ 
Public Health 19, $1201 \quad$ (2019). https://doi.org/10.1186/s12889-019-7540-4.

[10] UNAIDS (2012). UNAIDS report on the global AIDS epidemic. Available at http://www.unaids.org/sites/default/files/media_asse t/20121120_UNAIDS_Global_Report_2012_with_a nnexes_en_1.pdf.

[11]UNAIDS press release (2015). Success in reaching 15 by 15 shows that we can end the AIDS epidemic available at http://www.unaids.org/en/resources/pressce ntre/pressreleaseandstatementarchive/2015/ july/20150719_15x15_PR.

[12] Graham B, Daniel R, Gary WD, Jeanne E, Marina C, Natalie H, Jack W (2015). Investigating combination HIV prevention: isolated interventions or complex system JIAS open access. https://doi.org/10.7448/IAS.18.1.20499.

[13] UNAIDS (2015). Fast-tracking combination prevention towards reducing new HIV infection to fewer than 500,000 by 2020. http://www.unaids.org/sites/default/files/media_asse t/20151019_JC2766_Fasttracking_combination_prevention.pdf.

[14] UNAIDS (2017). Ending AIDS, progress towards the 90-90-90 targets. Available at http://www.unaids.org/sites/default/files/media_asse t/Global_AIDS_update_2017_en.pdf.

[15] UNAIDS (2018). Accelerating towards 90-9090. Available

at

http://www.unaids.org/en/resources/presscentre/feat urestories/2018/july/90-90-90-targets-workshop.

[16] UNAIDS (2018). 90-90-90 treatment target. Available at http://www.unaids.org/en/90-90-90.

[17] UNAIDS (2020): Press Release. Available at https://www.unaids.org/en/resources/presscentre/pre ssreleaseandstatementarchive/2020/july/20200706_g lobal-aids-report.

[18]UNAIDS (2015). Understanding fast-track, accelerating action to ends AIDS epidemic by 2030 . https://www.unaids.org/sites/default/files/media_ass et/201506_JC2743_Understanding_FastTrack_en.pd f.

[19] Sexual Partners, Partner Counseling and Referral Services (PCRS). Available at https://www.aids.gov/hiv-aids-basics/justdiagnosed-with-hiv-aids/talking-about-yourstatus/sexual-partners/.

[20]WHO; Call for case studies of HIV partner notification programmes. Available at http://www.who.int/hiv/mediacentre/news/hivpartner-notification/en/.

[21]WHO; Call for case studies of HIV partner notification programmes, http://www.who.int/hiv/mediacentre/news/hivpartner-notification/en/.

[22] Schneider G, Juday T, Wentworth C 3rd, Lanes S, Hebden T, Seekins D.(2013); Impact of health care payer type on HIV stage of illness at the time of initiation of antiretroviral therapy in the USA. AIDS Care. 2013;25(11):1470-6. DOI: 10.1080/09540121.2013.774316.

[23] Siegfried N, Uthman OA, Rutherford GW. (2017); Optimal time for initiation of antiretroviral therapy in asymptomatic, HIV-infected, treatmentnaive adults. Cochrane Database Syst Rev. 2010 March 17;(3): $\quad$ CD008272. DOI: 10.1002/14651858.CD008272.pub2, https://www.ncbi.nlms.nih.gov/pubmed/20238364. [24] Jennifer L. Weinberg and Carrie L. Kovarik, MD (2010); The WHO Clinical Staging System for HIV/AIDS, American Medical Association Journal Ethics, Volume 12, Number 3: 202-206.

[25]Federal Ministry of Health Abuja (2013). National HIV\&AIDS and Reproductive Health Survey (NARHS plus 2012). Available at https://naca.gov.ng/narhs-plus-2012-final/.

[26] Felix A. Ogbo, Andrew Mogaji, Pascal Ogeleka, Kingsley E. Agho, John Idoko, Terver Zua Tule, and Andrew (2017) Assessment of provider-initiated HIV screening in Nigeria with sub-Saharan African comparison. BMC Health Serv Res. 2017; 17: 188. DOI: 10.1186/s12913-017-2132-4.

[27] Adaora Adeline Okechukwu, Eno Ekop, Chinwendu Daniel Ndukwe, Kudirat Eyinade Olateju (2016). Acceptance of provider-initiated testing and counseling for HIV infection by caregivers in a tertiary health institution in Abuja, Nigeria: a crosssectional study. The Pan African Medical Journal. 2016;24:245. Doi:10.11604/pamj.2016.24.245.9057. 
[28] Nigerian HIV/AIDS Indicator and Impact Survey (2018); Technical Report.

[29] Moses Katbi, Adeoye Adegboye, Adefisayo Adedoyin, Fadimatu Yunusa, Gbenga Kayode, Maryam Bello, Patrick Dakum (2018). Effect of clients Strategic Index Case Testing on communitybased detection of HIV infections (STRICT study). International Journal of Infectious Diseases 74 (2018)
54-60.

DOI: https://doi.org/10.1016/j.ijid.2018.06.018.

[30] World Health Organization (2016): Guidelines on HIV Self-Testing and Partner Notification: Supplement to Consolidated Guidelines on HIV Testing Services. Accessed on October 2020 https://www.ncbi.nlm.nih.gov/books/NBK401684/. 